\title{
CThG1.pdf
}

\section{Photoacoustic tomography}

Lihong V. Wang, Ph.D.

Gene K. Beare Distinguished Professor

Optical Imaging Laboratory, Department of Biomedical Engineering

Washington University in St. Louis

Email: 1hwang@,biomed.wustl.edu; URL: http://oilab.seas.wustl.edu

\section{Summary (35 words)}

A pulsed laser produces a rapid small temperature rise in biological tissue, which leads to emission of ultrasonic waves due to thermoelastic expansion. The short-wavelength ultrasonic waves are then detected to form high-resolution tomographic images.

\section{Abstract}

The field of photoacoustic tomography has experienced considerable growth in the past few years. Although several commercially available pure optical imaging modalities, including confocal microscopy, two-photon microscopy, and optical coherence tomography, have been highly successful, none of these technologies can provide penetration beyond $\sim 1 \mathrm{~mm}$ into scattering biological tissues because they are based on ballistic and quasi-ballistic photons. Heretofore, there has been a void in high-resolution optical imaging beyond this depth limit. Photoacoustic tomography, which combines high ultrasonic resolution and strong optical contrast in a single modality, has filled this void. In this invited presentation, the fundamentals of photoacoustics are first introduced. Then, scanning photoacoustic microscopy and reconstruction-based photoacoustic tomography (or photoacoustic computed tomography) are covered. Recent progress are highlighted.

\section{Introduction}

Photoacoustic tomography (PAT) refers to imaging that is based on the photoacoustic effect. The motivation for PAT is to combine optical-absorption contrast with ultrasonic spatial resolution for deep imaging in the optical quasi-diffusive or diffusive regime, which is $>1 \mathrm{~mm}$ in most biological tissues. In PAT, the tissue is irradiated by usually a short-pulsed laser beam to achieve a thermal and acoustic impulse response. Locally absorbed light is converted into heat, which is further converted to a pressure rise via thermoelastic expansion. The initial pressure risedetermined by the volumetric specific optical absorption $\left(\mathrm{mJ} / \mathrm{cm}^{3}\right)$ and other thermal and mechanical properties - propagates as an ultrasonic wave, which is referred to as a photoacoustic wave. The photoacoustic wave is detected by an ultrasonic transducer, producing an electric signal. The electric signal is then amplified, digitized and transferred to a computer.

To form an image, a single-element ultrasonic transducer is scanned around the tissue; alternatively, an ultrasonic array can be used to acquire data in parallel. PAT has two major forms of implementation. One is based on a scanning focused ultrasonic transducer. Dark-field confocal photoacoustic microscopy belongs to this category. The other is based on an array of unfocused ultrasonic transducers in combination with a reconstruction algorithm.

\section{Image contrast}

The image contrast of PAT is based on optical absorption in the photoacoustic excitation phase. Selective optical absorption is associated with molecules such as oxygenated and deoxygenated 
hemoglobin and melanin. Concentrations of multiple chromophores whose spectra of absorption coefficient are different can be quantified simultaneously by varying the wavelength of the irradiating laser. Such quantification of oxygenated and deoxygenated hemoglobin, for example, can provide functional imaging of the total concentration and oxygen saturation of hemoglobin.

\section{Spatial resolution}

The spatial resolution of PAT is derived from ultrasonic detection in the photoacoustic emission phase. Due to strong optical scattering, pure optical imaging in biological tissue has either shallow imaging depth or low spatial resolution. Although light scattering broadens and attenuates light reaching an embedded object, the resulting ultrasonic signal provides better resolution than the optical signal in the optical quasi-diffusive or diffusive regime because ultrasonic scattering is 2-3 orders of magnitude weaker than optical scattering. The image resolution, as well as the maximum imaging depth, is scaleable with the ultrasonic frequency within the reach of diffuse photons. Specifically, as the ultrasonic center frequency and bandwidth increase, the spatial resolution improves at the expense of imaging depth because ultrasonic attenuation increases with frequency. In addition, PAT provides images devoid of speckle artifacts, which are conspicuous in both ultrasound and optical coherence tomography images.

\section{Sample image}

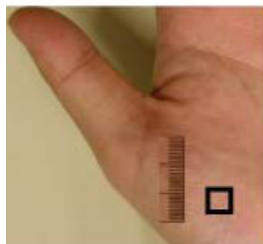

(a)

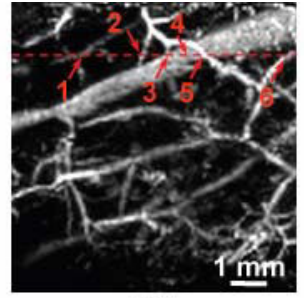

(b)

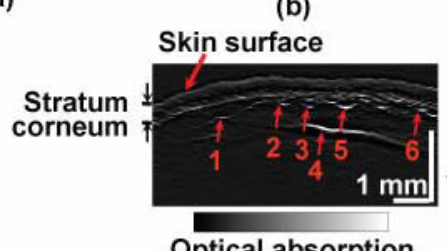

(c)

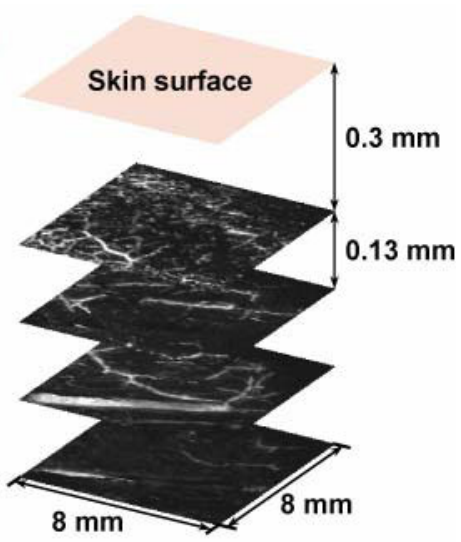

(d)

Figure 1. Imaging of the total hemoglobin concentration in subcutaneous vasculature of a human palm acquired in vivo by PAM at $584 \mathrm{~nm}$. (a) Photograph of the imaged area. (b) Maximum amplitude projection (MAP) image of the PA amplitude below the stratum-corneum layer projected along the $z$ axis. (c). B-scan image in the $z-x$ plane at the dashed line in panel $b$. The skin surface and the stratum corneum as well as blood vessels are visualized. Numbers 1-6 indicate the corresponding vessels in the MAP and B-scan images (d) A series of MAP images from different layers parallel with the skin surface. The first layer starts from $0.17 \mathrm{~mm}$ beneath the skin surface, whereas the layer spacing is $0.13 \mathrm{~mm}$. Reproduced with permission from [H. F. Zhang, K. Maslov, G. Stoica, and L. H. V. Wang, "Functional photoacoustic microscopy for high-resolution and noninvasive in vivo imaging," Nature Biotechnology, vol. 24, pp. 848-851, 2006.] 